Chrischta Ganz

\title{
Zweiter Kongress für Traditionelle Europäische Naturheilkunde: «Die Frau in der Naturheilkunde»
}

\section{Referate}

Namhafte Referentinnen und Referenten mit viel Praxiserfahrung boten den Teilnehmern ein spannendes und abwechslungsreiches Programm:

Doris Ritzmann eröffnete den ersten Kongresstag mit ihrem Referat «Endometriose - was ist das?». Endometriose ist bis heute eine Erkrankung mit vielen Fragezeichen geblieben, sowohl was ihre Ursachen als auch was ihre Behandlung betrifft. Der Name stammt von Endo (innerhalb) und Metra (Gebärmutter, Takt, Mitgefühl) ab. Was ist bei der Endometriose aus dem Takt gefallen? Die Endometriose ist als eine entzündlich-proliferative Krankheit zu interpretieren, als Dysregulation von Immunzellen, Zytokinen, Chemokinen und Enzymen im Blutplasma, in der Bauchhöhle, in den Eierstöcken sowie in der Gebärmutter und gehört in den Formenkreis der immunologischen Erkrankungen. Durch die vermehrte Produktion von Estradiol in den Endometriose-Zellen wird die Dysbalance noch verstärkt. Epithel- und Mesothel-Zellen verlieren so ihren Sozialkontakt und werden «Fibroblast-ähnlich». In dieser Form durchwachsen und vernarben sie gesundes Gewebe. Die Kommunikation zwischen Zellen und Organen ist dabei verändert. Ziel der Heilkunde ist es nun, Pflanzen (oder andere Behandlungsmethoden) zu finden, die «mitreden» können.

Chrischta Ganz stellte in ihrem Vortrag die Bedeutung der «Selbstheilungskräfte in der Frauenheilkunde»

Zum Thema «Die Frau in der Naturheilkunde» fand am 17. und 18. November 2016 im Alten Spital in Solothurn der zweite Kongress für Traditionelle Europäische Naturheilkunde (TEN) statt. Der Kongress wird vom Schweizerischen Verband der anerkannten Naturheilpraktikerinnen und Naturheilpraktiker (SVANAH) organisiert und stellt eine internationale Plattform für Vernetzung und Austausch für das Medizinalsystem der TEN und für die Heilmethoden, die in unserer Kultur verwurzelt sind, dar (Abb. 1).

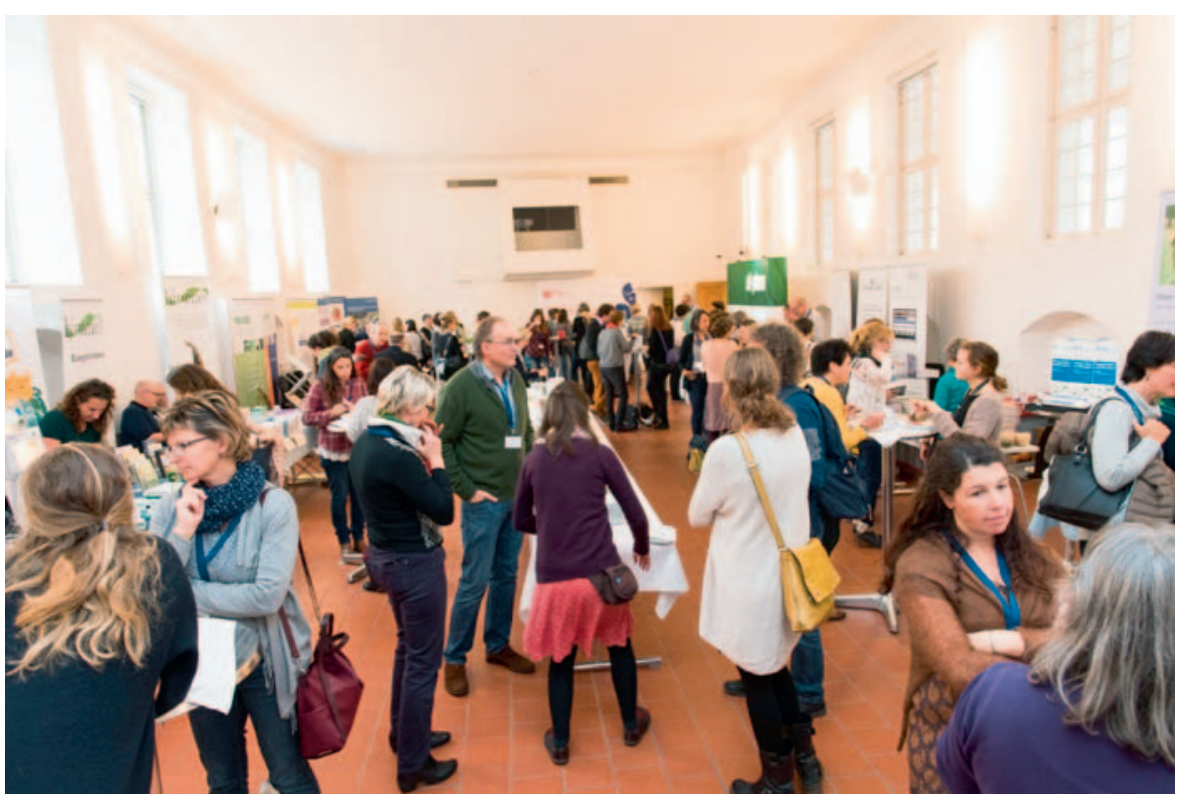

Abb. 1. Der TEN-Kongress bietet eine internationale Plattform für die Vernetzung und den Austausch von TEN-Fachleuten.

und effektvolle Methoden vor, um die Ressourcen der Patienten zur Bewältigung einer Erkrankung zu mobilisieren.

Verena Schönmann legte in ihrem Vortrag «Wechselvolle Jahre» den Fokus auf die weisen Aspekte der Wechseljahre und zeigte auf, wie wichtig es für Frauen in den Wechseljahren ist, unbekannte und überraschende Seiten willkommen zu heissen und anzuneh- men, um die innere und äussere Schönheit und Gesundheit zu pflegen und ein neues, inniges Verhältnis zum Körper zu finden.

Rosmarie Prica-Tönz eröffnete den Kursteilnehmern in ihrem Vortrag «Frauenheilkunde in der Hildegardmedizin» eine kurze historische Rückblende auf Hildegard von Bingen und den zeit- und sozialhistorischen Kontext, in dem sie gelebt und gewirkt hat.

\section{KARGER}

Fax +497614520714 (c) 2017 S. Karger GmbH, Freiburg
Chrischta Ganz 


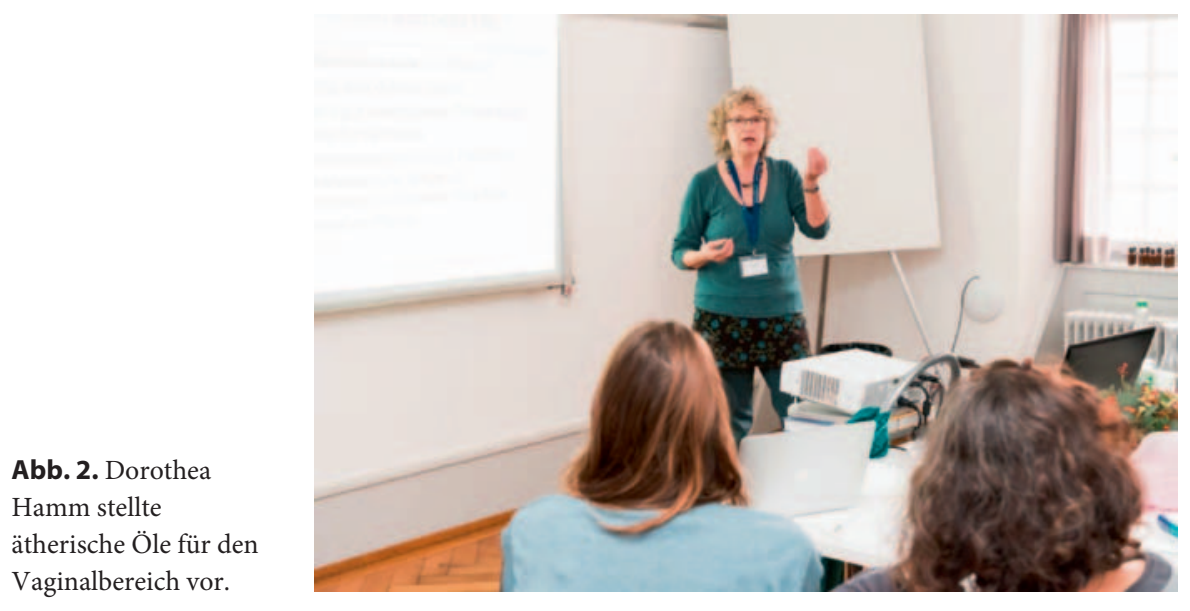

Kasten 1. Zwei Rezepturen

\begin{tabular}{|c|c|}
\hline \multicolumn{2}{|c|}{$\begin{array}{l}\text { Eine beliebte Mischung zur Vaginalschleim- } \\
\text { hautpflege, Behandlung von Hormonstörungen } \\
\text { und Vorbeugung von Vaginalinfektionen ist } \\
\text { folgende Rezeptur: }\end{array}$} \\
\hline \multicolumn{2}{|c|}{ Ölmischung bei Hormonstörungen: } \\
\hline Ylang-Ylang & $2 \mathrm{Tr}$ \\
\hline Sandelholz & $2 \operatorname{Tr}$. \\
\hline Muskatellersalbei & 2 Tr. \\
\hline Rosengeranie & $2 \operatorname{Tr}$. \\
\hline Bergamotte & $4 \mathrm{Tr}$. \\
\hline Basisöl (Jojoba- oder Ma & $20 \mathrm{ml}$ \\
\hline \multicolumn{2}{|c|}{$\begin{array}{l}\text { Anfänglich } 2 \times \text { täglich die Scheide einreib } \\
\text { später } 2 \times \text { wöchentlich. } \\
\text { Das Öl ist für Frauen jeden Alters geeignet. }\end{array}$} \\
\hline \multicolumn{2}{|c|}{$\begin{array}{l}\text { Ein weiteres Rezept, das Dorothea Hamm } \\
\text { den Kursteilnehmer/innen vorstellte, wird bei } \\
\text { Frauen nach einer Chemotherapie im Unterleib } \\
\text { verwendet. }\end{array}$} \\
\hline \multicolumn{2}{|l|}{ Strahlungsöl: } \\
\hline Niaouli & $20 \mathrm{Tr}$. \\
\hline Lavendel fein & 20 Tr. \\
\hline Basisöl (Johannisöl) & $100 \mathrm{ml}$ \\
\hline \multicolumn{2}{|c|}{$\begin{array}{l}\text { Bei empfindlicher Haut wird das Basisöl folgen- } \\
\text { dermassen verändert: }\end{array}$} \\
\hline Aloe-vera-Öl & $20 \mathrm{ml}$ \\
\hline Jojobaöl & $79 \mathrm{ml}$ \\
\hline Sanddornfruchtfleischöl & $1 \mathrm{ml}$ \\
\hline
\end{tabular}

Hildegard von Bingen hat ein riesiges systematisches, schriftliches Gesamtwerk hinterlassen. Ihre Medizin basiert auf der Humoralpathologie. So spielen in der Hildegardheilkunde die Ernährung, die humoralmedizinischen Qualitäten der einzelnen Nahrungsmittel und die Wirkung von Gewürzen und Heilpflanzen eine bedeutende Rolle.
Dorothea Hamm (Abb. 2) bot einen Workshop zum Thema «Ätherische Öle im Vaginalbereich» an und stellte verschiedene Rezepturen und Therapiekonzepte vor, die sie in ihrem Praxisalltag erfolgreich einsetzt (Kasten 1). Ätherische Öle - in ein gutes Pflanzenöl gemischt - pflegen und schützen die Schleimhäute. Sie wirken antibakteriell, antiviral und fungizid und können die Resistenzen von Bakterien auf Antibiotika reduzieren.

Iris Ritzmann-Wolff griff das Thema «Giftmischerinnen oder weise Frauen? Auf den Spuren weiblicher Heilkunde» auf. Anhand alter Kupferstiche und Zeichnungen zeigte sie die medizingeschichtliche Entwicklung der Heilberufe im Verlauf der letzten Jahrhunderte auf. Ritzmann teilte die Heilkundigen in drei Gruppen ein:

- Laien: Frauen, Mütter/Grossmütter, aber auch nebenberufliche Heilende wie Schäfer, Schmiede und Scharfrichter.

- Inoffizielle Heilkundige: Arzneihändler, Zahnbrecher, Stein- und Bruchschneider und Okulisten (von «Oculus», lat. "Auge»; diese führten vom Altertum bis in die frühe Neuzeit Starstiche als einfache Operationsmethode zur Behandlung des Grauen Stars aus).

- Offizielle Medizinalpersonen: Gelehrte Ärzte, Hebammen, handwerkliche Chirurgen, Wundärzte, Bader und Barbiere.
Frauen waren in allen drei Gruppen vertreten, insbesondere bei den Arzneihändlerinnen, Okulistinnen und Hebammen. Die Darstellungen auf verschiedenen alten Kupferstichen zeigten deutliche Analogien zu Hexendarstellungen.

Olaf Rippe baute in seinem Referat «Heilen im Einklang mit den Sternen - Das Mondmetall Silber und das Venusmetall Kupfer in der Frauenheilkunde» auf dem Gedankengut der Paracelsusmedizin auf (Abb. 3).

Heide Fischer erläuterte in ihrem Vortrag «Naturheilkundliche Behandlung bei HPV und Dysplasien», was den Patientinnen im Falle einer Dysplasie aus der naturheilkundlichen Sicht zu raten ist. Die Vorsorgeuntersuchung auf Zellveränderungen am Gebärmutterhals gehört seit den 1970er-Jahren zur gynäkologischen Routine. Auch die HPV-Impfung wurde kritisch beleuchtet.

Friedemann Garvelmann vertiefte das Wissen um die «Menstruation in Bezug auf humoralmedizinische Aspekte». Die Menstruationsblutung ist der natürliche Mechanismus des weiblichen Organismus zur Regulation des Wärmeprinzips. Je ausgeprägter das Wärmeprinzip einer Frau ist (konstitutionell oder aufgrund von Erkrankungen oder Lebenssituationen), umso wichtiger ist die Blutung und umso stärker pathogen wirkt deren Unterdrückung. So lassen sich auch die typischen Symptome im Klimakterium wie Hitzewallungen, Kongestion zum Kopf, Unruhezustände und übermässiges Schwitzen als typische Folge der ausbleibenden Menstruation im Sinne einer kompensatorischen Elimination des übersteigerten Wärmeprinzips erklären.

Sybille Binder zeigte in ihrem Vortrag «Ernährungsheilkunde bei Brusterkrankungen und Endometriose» ernährungsheilkundliche und ernährungswissenschaftliche Zusammenhänge zwischen Entzündung, Schmerz und Nahrung sowie Aspekte des Ernährungsverhaltens im Praxisalltag auf. Denn: Veränderungen im Zell- 
Abb. 3. Olaf Rippe beleuchtete in seinem Vortrag das Mondmetall Silber und das

Venusmetall Kupfer.

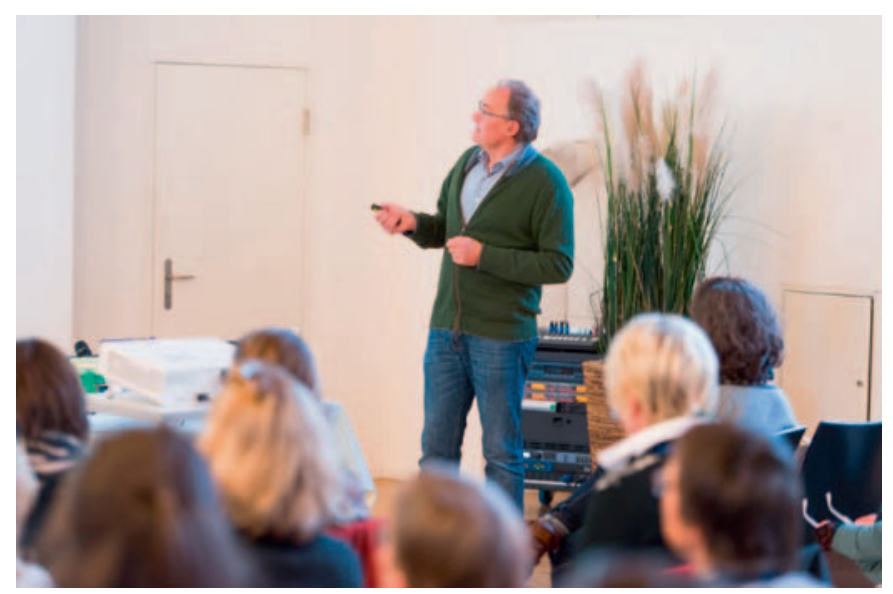

wachstum sowie Schmerzzustände und -qualitäten weisen eine enge Wechselwirkung mit dem auf, was und wie viel wir essen und trinken (Tab. 1).

Conwitha Lapke berichtete über die Rolle von «Mond und Saturn in der Spagyrik» und zeigte auf, wie die Frauenkraft individuell entfaltet werden kann.

Margret Madejsky erzählte in ihrem Referat vom «Garten der Aphrodite» und entführte die Teilnehmer zu den Heil- und Zauberpflanzen der Liebesgöttin. Mit den nach ihr benannten Aphrodisiaka werden die Sexualorgane gestärkt, die Sinnlichkeit geweckt und die Gefühlsintensität gesteigert.

Ausserdem leitete Margret Madejsky ein Seminar zum Thema «Natürliche Wege zum Wunschkind». Sie zeigte die vielfältigen Ursachen toxischer Belastung mit hormonartigen Umweltproblemstoffen und deren Folgeerkrankungen (Schilddrüsenfunktionsstörungen, Gelbkörperschwäche, Endometriose, Myome, seelische Blockaden) auf und stellte zahlreiche Naturheilmittel vor, welche die Entgif- tungsorgane entlasten, die hormonelle Balance wieder herstellen, den Zyklus regulieren, die Fruchtbarkeit fördern und die Frauengesundheit unabhängig vom Kinderwunsch stärken.

\section{Fazit}

Reichhaltiges Wissen und viele Anregungen konnten die Kongressteilnehmer aus den oben vorgestellten Referaten (und vielen weiteren Vorträgen) mit nach Hause nehmen. Der zweite TEN-Kongress stellte eine grosse Bereicherung für all jene Fachleute dar, die sich für die heimische Naturheilkunde interessieren und ihr Wissen vertiefen wollten.

\section{Ausblick}

Nach diesem spannenden und erfolgreichen Kongress lohnt sich der Ausblick auf den dritten TEN-Kongress, der vom 15.-17. November 2018 zum Thema «Kinder in der Naturheilkunde» stattfinden wird.
Tab. 1. Die wichtigsten entzündungsfördernden und entzündungslindernden Ernährungsaspekte

Entzündungsfördernde und schwächende Ernährungsaspekte

Zucker und raffinierte Kohlenhydrate, vor allem Mehlprodukte

Arachidonhaltige Nahrungsmittel wie Fleisch und Milchprodukte

Rotes Fleisch (Eisen)

Omega-6-Fettsäure-reiche Fette wie Sonnenblumen-, Distel- und Weizenkeimöl

Kaffee, Schwarztee, Alkohol

Verdeckte Allergien

Entgleisungen der Darmsymbiose Ständiges Essen und Naschen Unregelmässige Mahlzeitenverteilung Grosse Mengen Rohkost und rohes Obst Kühlschrankkalte Speisen und Getränke Künstliche Nahrungsbestandteile Tiefkühl- und Mikrowellenkost Ablenkung während dem Essen

Hastiges Essen, ungenügendes Kauen

Entzündungslindernde und stärkende Ernährungsmassnahmen

Genügend trinken

Viele sekundäre Pflanzeninhaltsstoffe

Omega-3-Fettsäure-reiche Nahrungsmittel wie fette Fische und Leinöl

Vitamin E (Pflanzenöle und Nüsse)

Magnesium

Vitamine der B-Gruppe

Verschiedene Spurenelemente

Rhythmische Mahlzeitenverteilung

Ausgewogenes Gleichgewicht von gekochten und rohen Nahrungsmitteln

Qualitativ hochwertige Grundprodukte

Saisonale Nahrungsmittel bevorzugen

Sich Zeit zum Essen nehmen, sich auf das Essen einlassen

Langsam essen und gut kauen

Möglichst alle Geschmacksrichtungen innerhalb einer Mahlzeit einsetzen

Mit Gewürzen und Kräutern Wärme und Dynamik des Verdauungsfeuers unterstützen

Abwechslungsreiche Nahrungsmittelauswahl

Nicht über die Sättigung hinaus essen 